\title{
A NOTE ON ISOTHERMAL NETS
}

\author{
V. G. GROVE
}

1. Introduction. It is the purpose of this paper to give a geometrical characterization of isothermally conjugate nets. This characterization is not dependent on the axis or ray curves of the net, and therefore not on the notation of conjugacy of the axis congruence nor on the ray congruence being harmonic to the sustaining surface. The characterization is made in terms of a property described by Bell [1] of a congruence being central to the surface $S$. A family of congruences associated with the tangent to a curve of the conjugate net is derived. The condition that the net be isothermally conjugate is expressed in terms of the property of any congruence of the family being central to $S$. The notion is extended to a characterization of isothermally orthogonal nets.

To fix the notation, let the coordinates $\left(x^{1}, x^{2}, x^{3}, x^{4}\right)$ of a generic point $x$ of the surface $S$ and the parameters $u, v$ be so chosen that they satisfy the following system of differential equations [3]

$$
x_{u u}=\theta_{u} x_{u}+\beta x_{v}+p x, \quad x_{v v}=\gamma x_{u}+\theta_{v} x_{v}+q x, \theta=\log R .
$$

The lines determined by the points $x_{u}, x_{v}$ and by the points $x, x_{u v}$ have been called the $R$-harmonic and $R$-conjugate lines respectively.

Let $r$ and $s$ be two points on the asymptotic tangents to the curves $v=$ const., $u=$ const. respectively. The coordinates $r, s$ of these points are given by the expressions

$$
r=x_{u}-b x, \quad s=x_{v}-a x .
$$

It is readily shown that as $x$ generates the curve $u=$ const. on $S$, the point $r$ generates a curve whose tangent is coplanar with the tangent to the locus of $s$ as $x$ generates $v=$ const. if and only if

$$
a_{u}-b_{v}=0 .
$$

The congruence $\Gamma_{2}$ of lines $(r, s)$ is therefore central to $S$ if and only if (1.3) is satisfied. If $\Gamma_{2}$ is central to $S$, then the curves corresponding to the developables of $\Gamma_{2}$ either form a conjugate net, coincide with asymptotic curves, or are indeterminate [5]. A similar statement holds for the reciprocal congruence $\Gamma_{1}$ of $\Gamma_{2}$.

2. Isothermally conjugate nets. Let the differential equation of a

Presented to the Society, September 1, 1949; received by the editors July 3, 1949.

${ }^{1}$ Numbers in brackets refer to the references cited at the end of the paper. 
one-parameter family of curves $C_{\lambda}$ on $S$ be

$$
M d u-N d v=0, \quad \lambda=M / N,
$$

this family being neither of the families of asymptotic curves. Let $y$ be a point on the tangent to that curve of $C_{\lambda}$ through $x$, but not on the $R$-harmonic line. The coordinates of $y$ have the form

$$
y=S x+N x_{u}+M x_{v}, \quad M N S \neq 0 .
$$

The projections $r, s$ of $y$ from the points $x_{v}$ and $x_{u}$ on the asymptotic tangents have coordinates given by the expressions

$$
r=x_{u}+S x / N, \quad s=x_{v}+S x / M .
$$

We have called these points the integral generating points [4]. However, in this paper we do not insist that they be invariant.

The tangent to the locus of $s(r)$ as $x$ generates the curve $v=$ const. ( $u=$ const.) intersects the plane determined by the tangent to $u=$ const. ( $v=$ const.) and the $R$-conjugate line in a point whose projection from $x_{u v}$ on the tangent plane is the point $\rho(\sigma)$ whose coordinates are given by the expression [4]

$$
\rho=x_{u}+\frac{\partial}{\partial u} \log \left(\frac{S}{M}\right) x, \quad \sigma=x_{v}+\frac{\partial}{\partial v} \log \left(\frac{S}{N}\right) x .
$$

If we define points $\rho_{h}, \sigma_{h}$ so that one of the cross ratios of $x, x_{u}, \rho, \rho_{h}$, and of $x, x_{v}, \sigma, \sigma_{h}$, is $h$, the coordinates of these points are given by

$$
\rho_{h}=x_{u}+h \frac{\partial}{\partial u} \log \frac{S}{M} x, \quad \sigma_{h}=x_{v}+h \frac{\partial}{\partial v} \log \frac{S}{N} x .
$$

We shall call these points the differential generating points, and the line determined by them the differential generating line. A similar appellation will be used for the congruence of these lines.

From (1.3) it follows that the differential generating congruence determined by (2.4) is central to $S$ if and only if

$$
\frac{\partial^{2}}{\partial u \partial v} \log \left(\frac{M}{N}\right)=0
$$

that is, if and only if the conjugate net

$$
M^{2} d u^{2}-N^{2} d v^{2}=0
$$

is isothermally conjugate.

We shall say that the curves (2.1) have the isothermal property if (2.5) is satisfied. Hence if one family of curves of a conjugate net has 
the isothermal property, the other has, and all curves of the pencil of conjugate nets whose base net is the given net have the property. An immediate application of this notion gives the following theorem. $A$ surface is isothermally asymptotic if and only if any curve of the Darboux-Segre pencil of conjugate nets has the isothermal property.

3. Conclusion. Suppose a given conjugate net defined by (2.6) is isothermally conjugate. By a transformation of independent variables, we may make $M=N$. Equations (2.4) may be written in the form

$$
\rho_{h}=x_{u}-\frac{\partial}{\partial u} \log A x, \quad \sigma_{h}=x_{v}-\frac{\partial}{\partial v} \log A x
$$

wherein

$$
A=(M / S)^{h} .
$$

The curves on $S$ corresponding to the developables of the differential generating congruence [5] are defined by the differential equation

$$
P d u^{2}-Q d v^{2}=0 \text {, }
$$

wherein

$$
\begin{aligned}
& P=A_{u u}-\left(\theta_{u} A_{u}+\beta A_{v}+p A\right), \\
& Q=A_{v v}-\left(\gamma A_{u}+\theta_{v} A_{v}+q A\right) .
\end{aligned}
$$

It follows that any solution A of (1.1) gives a congruence of lines $\Gamma_{2}$ whose developables are indeterminate.

Similarly the developables of the reciprocal congruence $\Gamma_{1}$ of $\Gamma_{2}$ with respect to the quadrices of Darboux are indeterminate if and only if $A$ is a solution of

$$
\begin{aligned}
& A_{u u}=\theta_{u} A_{u}-\beta A_{v}+\left(p+\beta \theta_{v}+\beta_{v}\right) A, \\
& A_{v v}=-\gamma A_{u}+\theta_{v} A_{v}+\left(q+\gamma \theta_{u}+\gamma_{u}\right) A .
\end{aligned}
$$

If both congruences $\Gamma_{1}, \Gamma_{2}$ have indeterminate developables, $S$ is necessarily isothermally asymptotic.

We now extend the characterization of isothermal conjugacy of a net to a characterization of isothermally orthogonal nets. Let the surface be referred to the orthogonal net as parametric. Let a oneparameter family of curves be given by (2.1). The coordinates $y^{i}(i=1,2,3)$ of any point $y$ on the tangent to this curve have the form

$$
y^{i}=x^{i}+\left(N x_{u}^{i}+M x_{v}^{i}\right) / S, \quad i=1,2,3 .
$$


The orthogonal projections $r, s$ of $y$ on the tangents to the curve of the net have coordinates given by the expressions

$$
r^{i}=x^{i}+\frac{N}{S} x_{u}^{i}, \quad S^{i}=x^{i}+\frac{M}{S} x_{v}^{i}
$$

As $x$ generates the curve $v=$ const., $s$ generates a curve whose tangent has the following direction numbers

$$
s_{u}^{i}=x_{u}^{i}+\left(\frac{M}{S}\right){ }_{u}^{i} x_{v}+\frac{M}{S} x_{u v}^{i}
$$

This tangent intersects the plane determined by the tangent to $v=$ const. and Green's $R$-reciprocal line [2] of the ideal line in the tangent plane in the point whose coordinates are

$$
x^{i}-\lambda\left(x_{u}^{i}+\frac{M}{S} x_{u v}^{i}\right), \quad \frac{1}{\lambda}=\frac{\partial}{\partial u} \log \left(\frac{M}{S}\right) .
$$

If we project on the tangent plane to $S$ this latter point by a line parallel to this $R$-reciprocal, a point $\rho$ is found whose coordinates are given by the expression

$$
\rho^{i}=x^{i}-\lambda x_{u}^{i}
$$

In a similar manner a point $\sigma$ whose coordinates $\sigma^{i}$ are given by the expression

$$
\sigma^{i}=x^{i}-\mu x_{v}^{i}, \quad \frac{1}{\mu}=\frac{\partial}{\partial v} \log \left(\frac{N}{S}\right)
$$

may be defined. We shall say that the congruence of lines $(\rho \sigma)$ is central to $S$ with respect to the given orthogonal parametric net if the tangent to the locus of $\rho$ as $x$ generates $u=$ const. intersects the tangent to the locus of $\sigma$ as $x$ generates $v=$ const. The congruence of lines $(\rho \sigma)$ is therefore central to $S$ with respect to the given orthogonal net if and only if

$$
\frac{\partial^{2}}{\partial u \partial v} \log \left(\frac{M}{N}\right)=0
$$

The net whose differential equation is

$$
E d u^{2}-G d v^{2}=0
$$

is uniquely determined as that orthogonal net the tangents to whose curves separate the tangents to the curves of the given orthogonal 
net harmonically. If the points $\rho, \sigma$ defined by (3.4), (3.5) are defined using either one of the two families of curves of (3.7), it follows readily that the congruence of such lines $(\rho \sigma)$ is central to $S$ with respect to the given orthogonal net if, and only if, the net is isothermally orthogonal.

As a final application of the notions in this paper we derive by geometrical methods all of the classical canonical lines except the projective normal. Suppose that the differential equations (1.1) are in the Fubini canonical form, that is, suppose $R=\beta \gamma$. Suppose that the line determined by the integral invariant points defined by (2.2) coincides with Bell's $R_{\lambda}$-associate [1] of the reciprocal of the projective normal for $\lambda=M / N$. It follows that

$$
\frac{S}{M}=\gamma \frac{M}{N}, \quad \frac{S}{N}=\beta \frac{N}{M} .
$$

It follows that the curve $C_{\lambda}$ is any one of the curves of Segre. For these values of $M / N$ the differential generating line determined by (2.3) is determined by

$$
\rho_{h}=x_{u}+\frac{h}{3} \phi x, \quad \sigma_{h}=x_{v}+\frac{h}{3} \psi x,
$$

wherein as usual

$$
\phi=\frac{\partial}{\partial u} \log \left(\beta \gamma^{2}\right), \quad \psi=\frac{\partial}{\partial v} \log \left(\beta^{2} \gamma\right) .
$$

That is if the integral generating line is the $R_{\lambda}$-associate of the reciprocal of the projective normal, then the curve $C_{\lambda}$ is a curve of Segre and the differential generating line is any one whatever of the classical canonical lines of the second kind.

\section{REFERENCES}

1. P. O. Bell, $A$ study of curved surfaces by means of certain associated ruled surfaces, Trans. Amer. Math. Soc. vol. 46 (1939) pp. 389-409.

2. G. M. Green, Memoir on the general theory of surfaces and rectilinear congruences, Trans. Amer. Math. Soc. vol. 20 (1919) pp. 80-153.

3. V. G. Grove, On canonical forms of differential equations, Bull. Amer. Math. Soc. vol. 36 (1930) pp. 582-586.

4. - Generalized canonical lines, Duke Math. J. vol. 15 (1948) pp. 95-103.

5. E. P. Lane, $A$ treatise on projective differential geometry, The University of Chicago Press, 1942.

Michigan State College 